\title{
$\Rightarrow$ VIROLOGY
}

\section{A novel state of undress}

DOI:

10.1038/nrmicro1434

URLs

UniProtKB: http://ca.expasy. org/sprot

A34: http://ca.expasy.org/ uniprot/P21057 B5: http://ca.expasy.org/ uniprot/P21115
Enveloped viruses penetrate target cells by fusing their single lipid membrane with the target cell membrane and releasing the infective naked viral core. But some virus forms such as the extracellular enveloped virus (EEV) of Vaccinia virus have two lipid membranes, and therefore cell entry requires the removal of both lipid barriers. New work from Geoffrey Smith's laboratory at Imperial College London has uncovered a unique way in which EEV sheds its outer lipid membrane, providing novel insights into virus entry and also a new target for antiviral therapy.

The authors studied the binding and entry of EEV to cells by immuno-electron microscopy and observed that the outer EEV membrane ruptures at the site of cell contact. This disruption, however, takes place in the absence of membrane fusion and the shed EEV outer membrane remains draped over the underlying single-enveloped virion, which is called an intracellular mature virus (IMV). The single membrane of IMV subsequently fuses with the target cell membrane and the virus core enters the cell.

Because disruption of the EEV outer membrane takes place only on contact with a cell, and not a synthetic substrate, the authors proposed that outer membrane dissolution required the interaction between ligands on the virus and the target cell surface. A series of experiments identified the virus glycoproteins A34 and $\mathrm{B} 5$, and cellular surface polyanions, known as glycosaminoglycans, as the required ligands. This is the first example of the removal of a virus membrane without fusion, which Smith and colleagues term ligand- dependent non-fusogenic viral membrane dissolution.

The authors went on to show that a soluble polyanion such as heparin can be used to rupture the EEV outer envelope in vitro, exposing the IMV and allowing neutralization by anti-IMV monoclonal antibodies. The combined administration of polyanions and anti-IMV antibodies protected mice against disease with Vaccinia virus, demonstrating the therapeutic potential of this strategy.

The EEV virion is the form of Vaccinia virus that spreads within the host and therefore an increased understanding of its entry mechanism and the identification of a strategy to target EEV entry are important advances in this field.

Shannon Amoils

ORIGINAL RESEARCH PAPER Law, M. et al.

Ligand-induced and nonfusogenic dissolution of a viral membrane. Proc. Natl Acad. Sci. USA 11, 5989-5994 (2006)

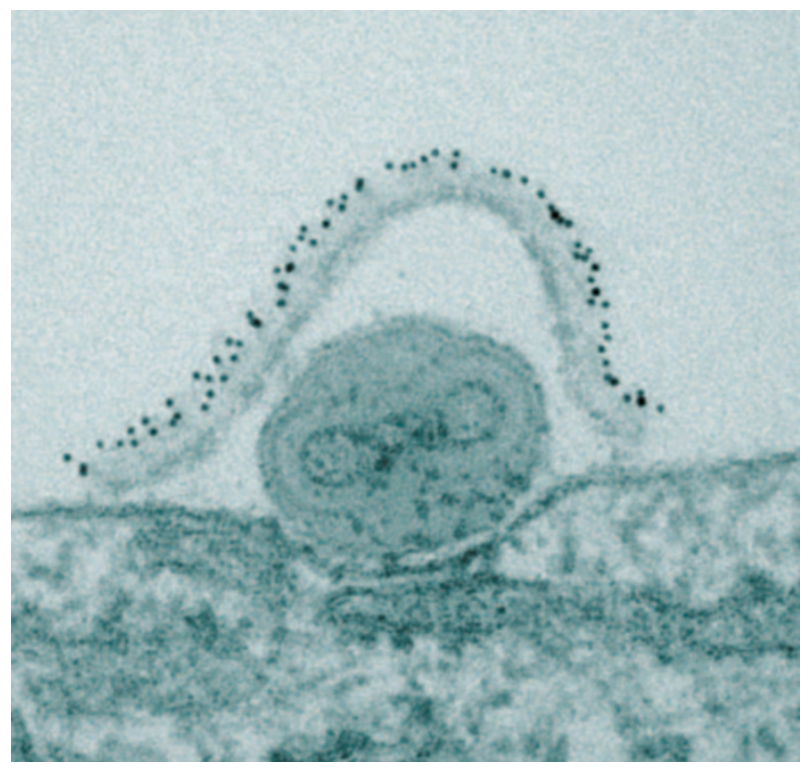

Immuno-electron microscopy showing non-fusogenic disruption of the EEV outer membrane, which exposes the IMV particle to the cell surface. Image reproduced with permission from Law et al. @ (2006) National Academy of Sciences, USA. 